\title{
The epidemiology of postnatal depression in Ethiopia: a systematic review and meta-analysis
}

Bereket Duko ${ }^{1,2^{*}}$ (D) Dereje Wolde ${ }^{3}$ and Yonas Alemayehu ${ }^{1}$

\begin{abstract}
Background: Postnatal depression is among the common mental health problems that occur during the postnatal period. However, it is left undiagnosed in low- and middle-income countries including Ethiopia. Therefore, this systematic review and meta-analysis aimed to systematically summarize the available evidence on the epidemiology of postnatal depression in Ethiopia and suggest recommendations for future clinical practice.

Methods: The Preferred Reporting Items for Systematic Reviews and Meta-Analyses (PRISMA) guideline was followed to conduct this systematic review and meta-analysis. We searched PubMed, SCOPUS, EMBASE and Google Scholar databases for the relevant articles that assessed the prevalence of postnatal depression in Ethiopia. We used a random-effect model to conduct a meta-analysis. We conducted a subgroup and sensitivity analysis to explore the source of heterogeneity. Cochrane Q- and the $\mathrm{I}^{2}$-test were used to check the heterogeneity of the included studies. The presence of publication bias was also checked by visual inspection of symmetry and Egger's test.

Results: The pooled estimated prevalence of postnatal depression in Ethiopia was $20.1 \%$ (95\% Cl 12.7-30.2). The pooled prevalence of postnatal depression in the studies that were conducted in community settings and used the Patient Health Questionnaire to assess postnatal depression [16.6\% (95\% Cl 8.90-28.99)] was lower than the prevalence in studies based in institutions and that used the Edinburgh Postnatal Depression Scale $[23.2 \%(95 \% \mathrm{Cl}$ 14.50-28.5)]. Further, in a leave-one-out sensitivity analysis the prevalence of postnatal depression ranges between $15.4 \%$ and $25.4 \%$. Unplanned pregnancy $[A O R=3.46,95 \% \mathrm{Cl}(2.37-5.04)]$, age between $15-24$ years $[\mathrm{AOR}=1.72,95 \%$ $\mathrm{Cl}(1.11-2.68)]$, marital problems $[\mathrm{AOR}=3.07,95 \% \mathrm{Cl}(2.36-3.99)]$, experiencing the death of infant $[\mathrm{AOR}=3.41,95 \% \mathrm{Cl}$ (1.91-6.09)] and history of substance use $[A O R=3.47,95 \% \mathrm{Cl}(2.17-5.56)]$ were associated with the increased odds of postnatal depression in Ethiopia.
\end{abstract}

Conclusion: The prevalence of postnatal depression in Ethiopia was high. Therefore, the concerned body should give due attention to improve reproductive health services through early detection of risk factors of postnatal depression.

Keywords: Postnatal depression, Epidemiology, Associated factors, Ethiopia

*Correspondence: berkole.dad@gmail.com

${ }^{1}$ Faculty of Heath Sciences, College of Medicine and Health Sciences, Hawassa University, Hawassa, Ethiopia

Full list of author information is available at the end of the article

\section{Background}

The postpartum period is a challenging phase characterized by significant biological, physical, social change, and also with an increased risk of postnatal depression (PND) [1]. PND can be explained by the presence of various groups of depressive symptoms and syndromes which prevail within the first 12 months of childbirth [2,3]. Numerous signs and original author(s) and the source, provide a link to the Creative Commons licence, and indicate if changes were made. The images or other third party material in this article are included in the article's Creative Commons licence, unless indicated otherwise in a credit line to the material. If material is not included in the article's Creative Commons licence and your intended use is not permitted by statutory regulation or exceeds the permitted use, you will need to obtain permission directly from the copyright holder. To view a copy of this licence, visit http://creativecommons.org/licenses/by/4.0/. The Creative Commons Public Domain Dedication waiver (http://creativeco mmons.org/publicdomain/zero/1.0/) applies to the data made available in this article, unless otherwise stated in a credit line to the data. 
symptoms such as loss of interest in daily activities, loss of appetite, disturbed sleep, decreased concentration, and negative attitude toward new baby, suicidal ideation, guilty feeling, and easily fatigability define the presence of PND [3, 4].

Reports from the World Health Organization (WHO) in 2017 suggested that more than 322 million people were suffered from depression [5] and out of this, approximately 29.9 million people were from Africa. The Global Burden of Diseases study in 2015 systematically analyzed the data of 17 lowand middle-income countries (LMICs) and reported an $18.4 \%$ prevalence of PND [6]. Previous systematic review and meta-analysis conducted in 2012 to assess the pooled prevalence of perinatal common mental disorders including depression in LMICs reported $19.8 \%$ [7]. Further, studies conducted in African countries such as South Africa and Ethiopia reported that the prevalence of postnatal depression ranged between 30 and $50 \%$, suggesting one in every ten women in the postnatal period experiences depression [8-11].

A significant number of women are at higher risk of developing depression in the postnatal period due to different risk factors. The most common risk factors has been reported in the previous studies include domestic violence [12], younger age [13, 14], lowincome [15], lack of social support [16], unplanned pregnancy [15-18], anxiety during pregnancy, stressful recent life events, low education, past history of mental illness including depression and history of abortion [19-22].

Women who suffer from postnatal depression may have problems with daily functioning, and may also be at increased risk for suicidal thoughts and behaviors [6]. This can also result in poor mother to infant relationships, infectious illness to infants, and affect children's growth and development [23]. Besides, it could result in emotional, behavioral, and cognitive problems in the later life of the child [23, 24].

Although postnatal depression is treatable and clinical as well as behavioral interventions can be delivered by specially-trained non-specialists, there is limited evidence about the prevalence and related risk factors for postnatal depression in Ethiopia. Complementing this, a study conducted in 2016 revealed a lack of evidence on the prevalence of pre-and postnatal mental health problems in low-and-middle-income countries, suggesting the urgency of pooled estimated data on the topic to inform policymakers to address the disease burden [25]. Therefore, this systematic review and meta-analysis aimed to systematically summarize the available evidence on the epidemiology of postnatal depression in Ethiopia.

\section{Methods}

\section{Research design}

The Preferred Reporting Items for Systematic Reviews and Meta-Analyses (PRISMA) guideline was followed to conduct this systematic review and meta-analysis [26]. The review was conducted using a predesigned protocol for database searching, data extraction, inclusion-exclusion criteria, quality evaluation, data synthesis, and analysis (Additional file 1).

\section{Search strategy and selection process}

We searched PubMed, SCOPUS, and EMBASE databases for the possible relevant articles that assessed the prevalence of postnatal depression in Ethiopia using the following terms and keywords: (epidemiology OR prevalence OR magnitude) AND (perinatal depression OR postnatal depression OR maternal depression OR postpartum depression) AND Ethiopia. Furthermore, we repeated searching using the following search terms and keywords: (perinatal depression OR postnatal depression OR maternal depression OR postpartum depression) AND (associated factors OR correlates OR risk factors OR determinants) AND Ethiopia. We also searched EMBASE and SCOPUS using database-specific subject headings. Besides, we scanned the reference lists of the included literature.

\section{Eligibility criteria}

Our review included the observational studies that assessed the prevalence and associated factors of postnatal depression in Ethiopia. We have included all the studies irrespective of the publication date. Commentaries, editorials, reviews, and letters to editors were excluded from the review.

\section{Methods for data extraction and quality assessment}

The review was guided by the Preferred Reporting Items for Systematic Review and Meta-Analysis (PRISMA) [27]. The data extraction was performed by two reviewers (BD and MT) independently. We utilized a predefined data extraction form to extract data. The authors' name, year of publication, the sample size, study design, study setting, and the instrument used to assess postnatal depression, as well as associated factors along with adjusted odds ratios, were extracted from each study. The Newcastle-Ottawa Scale (NOS) which is adapted for cross-sectional studies was used to check the quality of studies included in the review [28]. The tool used to assess postnatal depression, the quality of the methods, sample size, sample representativeness, and comparability between participants were the domains NOS scale to check the quality of studies included in the review. The agreement between the evaluators was 
appraised using agreement beyond chance (unweighted kappa) (BD and DW). The levels of poor, slight, fair, moderate, substantial, and very high levels of agreement were depicted by the values $0,01-0.02,0.021-0.04,0.041-$ $0.06,0.061-0.08$, and $0.081-1.00$, respectively [29].

\section{Data synthesis and analysis}

We conducted a meta-analysis using the Comprehensive Meta-Analysis software version 3.0. The random-effect model was used to pool the overall prevalence of postnatal depression in Ethiopia [30]. The Cochrane Q-and the $\mathrm{I}^{2}$-statistics were used to assess the heterogeneity between the studies [31]. The values of 25,50 , and $75 \%$ represented a low, medium, and high level of heterogeneity respectively [32]. We have also conducted the subgroup and sensitivity analysis to assess the possible source of heterogeneity. Publication bias was evaluated by using Egger's test and visual inspection of the symmetry in funnel plots. The level of significance was set at $\mathrm{P}<0.05$.

\section{Results}

\section{Identification of studies}

A total of 272 articles was identified both on an electronic database and manual search. We excluded 255 publications during the review of titles, duplicates, and abstracts as they did not fulfill the eligibility criteria (Fig. 1). Therefore, 17 full-text articles were included for further screening and 12 of these were excluded. Finally, five full-text articles were included in the systematic review and meta-analysis.

\section{Characteristics of included studies}

Table 1 depicted the characteristics of the included studies. A total of 4751 study participants were included in the current review. The studies included in the review were conducted between 2014 and 2017. All studies included in the current systematic review and metaanalysis used a cross-sectional study design. Out of these, three studies were institution-based cross-sectional whereas two studies were community-based studies. Three studies were conducted in South Nation Nationalities and the People Regional States (SNNPRS), one was conducted in the Amhara region and one was conducted in Addis Ababa. Three studies used the Edinburgh Postnatal Depression Scale (EPDS) and two studies used the Patient Health Questionnaire (PHQ9) to assess postnatal depression.

\section{The quality of the included studies}

The Newcastle-Ottawa Scale (NOS) which is adapted for cross-sectional studies was used to check the quality of studies included in the review. The quality evaluation of the included studies based on the NOS revealed that three studies were of high methodologic quality whereas two studies were of moderate quality. The agreed levels between the authors regarding the quality of the studies included the meta-analysis ranged from moderate to very high levels of agreement (Table 1).

\section{A meta-analysis of the prevalence of postnatal depression in Ethiopia}

The overall pooled prevalence estimate of postnatal depression in Ethiopia was $20.1 \%$ (95\% CI 12.7-30.2) and the observed heterogeneity was significant $\left(I^{2}=99.408 \%\right.$; $\mathrm{Q}=154.300, \mathrm{df}=4, \mathrm{p}<0.001)($ Fig. 2).

\section{Subgroup and sensitivity analysis}

We performed subgroup and sensitivity analysis to identify the source of heterogeneity among the studies. The pooled prevalence of postnatal depression in the studies that were conducted in community settings and used the Patient Health Questionnaire to assess postnatal depression [16.6\% (95\% CI 8.90-28.99)] was lower than the prevalence in studies based in institutions and that used the Edinburgh Postnatal Depression Scale [23.2\% (95\%CI 14.50-28.5)] (Fig. 3). The heterogeneity was significant in both studies, that were conducted in the community and institution-level $\left(I^{2}=97.085 ; \mathrm{Q}=34.303, \mathrm{df}=1\right.$, $\mathrm{p}<0.001)$ and $\left(I^{2}=91.971 ; \mathrm{Q}=24.909, \mathrm{df}=2, \mathrm{p}<0.001\right)$ respectively.

Furthermore, we conducted a leave-one-out sensitivity analysis to identify the possible source of heterogeneity in the pooled meta-analysis of the prevalence of postnatal depression in Ethiopia. This analysis showed that the overall prevalence was strong and not dependent on a single study. The pooled prevalence of postnatal depression ranges between $15.4 \%(14.3-16.5 \%)$ and $25.4 \%$ (23.3-27.6\%) (Table 2).

\section{Statistical analysis of factors associated with postnatal depression}

In the random effect model, we identified the following determinants or risk factors of PND. The odds of developing PND was higher among women who did not plan their pregnancy $[\mathrm{AOR}=3.46,95 \% \mathrm{CI}(2.37-5.04)]$. Women aged between 15 and 24 years were more likely to report symptoms of PND when compared to the older one $[\mathrm{AOR}=1.72,95 \% \mathrm{CI}(1.11-2.68)]$. Women who reported marital problems had an increased odds of PND $[\mathrm{AOR}=3.07,95 \% \mathrm{CI}(2.36-3.99)]$. Further, women experiencing the death of infant $[\mathrm{AOR}=3.41,95 \% \mathrm{CI}(1.91-$ 6.09)] and reported a history of substance use such as alcohol and Khat $[\mathrm{AOR}=3.47,95 \% \mathrm{CI}(2.17-5.56)]$ were more likely to develop PND when compared to non-use. Moreover, the findings of this review also showed that 


\section{Identifications}

Screening
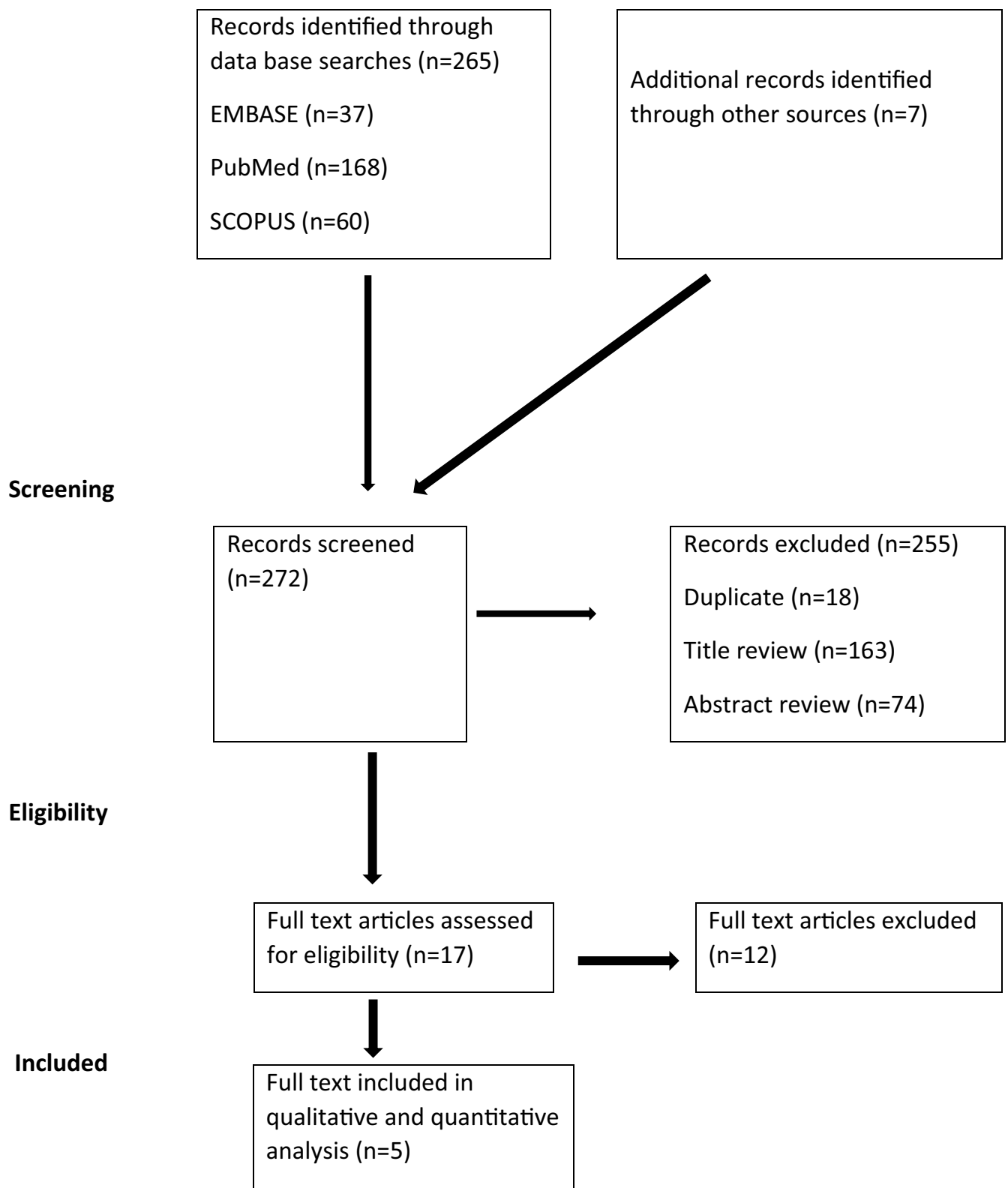

Fig. 1 PRISMA flowchart of review search

getting low-income as explained by perceived financial hardship and experienced hunger in the past months significantly increased the odds of PND [AOR $=2.42,95 \%$ CI (1.66-3.53)]. Poor social support, history of previous depression, domestic violence, and grand multiparty, perinatal complications, experiencing hunger in the preceding 1 month, stressful events, and history of abortion were factors significantly associated with postnatal depression in the respective studies not consistently.

\section{Publication bias}

Publication bias may occurs in the systematic review and meta-analysis when the results of published studies are potentially different from the results of unpublished studies. The bias can represent all forms of bias such as outcome-reporting bias, gray-literature bias, time-lag bias, full-publication bias, citation bias, language bias as well as media-attention bias. This can be checked by the visual inspection of the funnel plot and Egger's regression test. 
Table 1 Characteristics of included studies: epidemiology of postnatal depression in Ethiopia

\begin{tabular}{lllllllr}
\hline Author name, year & Region & Sample size & Study design & Settings & $\begin{array}{l}\text { Data } \\
\text { collection } \\
\text { tool }\end{array}$ & $\begin{array}{l}\text { Prevalence (\%) } \\
\text { NOS }\end{array}$ \\
& & & & & & & \\
\hline Kereie et al. [11] & SNNPRS & 408 & Cross-sectional & Institution-based & EPDS & 33.82 & 7 \\
Fantahun et al. [18] & Addis Ababa & 618 & Cross-sectional & Institution-based & EPDS & 23.30 & 9 \\
Toru et al. [14] & SNNPRS & 456 & Cross-sectional & Community-based & PHQ-9 & 22.40 & 8 \\
Shewangizaw et al. [19] & Amhara & 122 & Cross-sectional & Institution-based & EPDS & 13.11 & 7 \\
Azale et al. [20] & SNNPRS & 3147 & Cross-sectional & Community-based & PHQ-9 & 12.2 & 9 \\
\hline
\end{tabular}

PHQ-9 Patient Health Questionnaire, EPDS Edinburgh Postnatal Depression Scale

Study name

Kereie et al, 2018

Fantahun et al, 2018

Toruet al, 2018

Shewangizaw et al, 2018

Azale et al, 2018

\section{Statistics for each study}

Event rate and $95 \% \mathrm{Cl}$
Event Lower Upper

rate limit limit $p$-Value
$0.233 \quad 0.201 \quad 0.268$

$0.224 \quad 0.188$

0.265

0.203

0.134

0.302

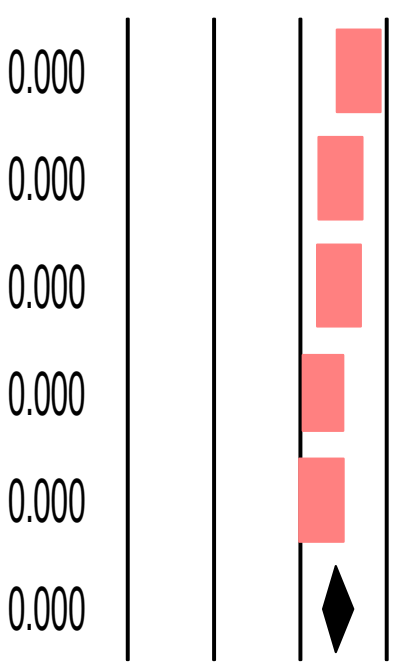

$-1.00 \quad-0.50 \quad 0.00 \quad 0.50 \quad 1.00$

Fig. 2 The prevalence of postnatal depression in Ethiopia: a meta-analysis

In this systematic review, both Egger's regression test and visual inspection of the funnel plot, revealed no evidence of significant publication bias $((\mathrm{B}=6.98, \mathrm{SE}=6.51$, $\mathrm{P}=0.36)$ (Fig. 4).

\section{Discussion}

This systematic review and meta-analysis included five studies with a total of 4751 study participants. The pooled estimated prevalence of postnatal depression in Ethiopia was $20.1 \%$. Unplanned pregnancy, age between
15 and 24 years, marital problems, experiencing the death of the infant, history of substance use and having lower income were identified factors that were found to increase the odds of postnatal depression. Three studies were conducted in South Nation Nationalities and the People Regional States (SNNPRS), one from each was conducted in the Amhara region and Addis Ababa and produced inconsistent rates of prevalence. Therefore, the findings of this systematic review and meta-analysis are very important to guide postnatal clinical services 


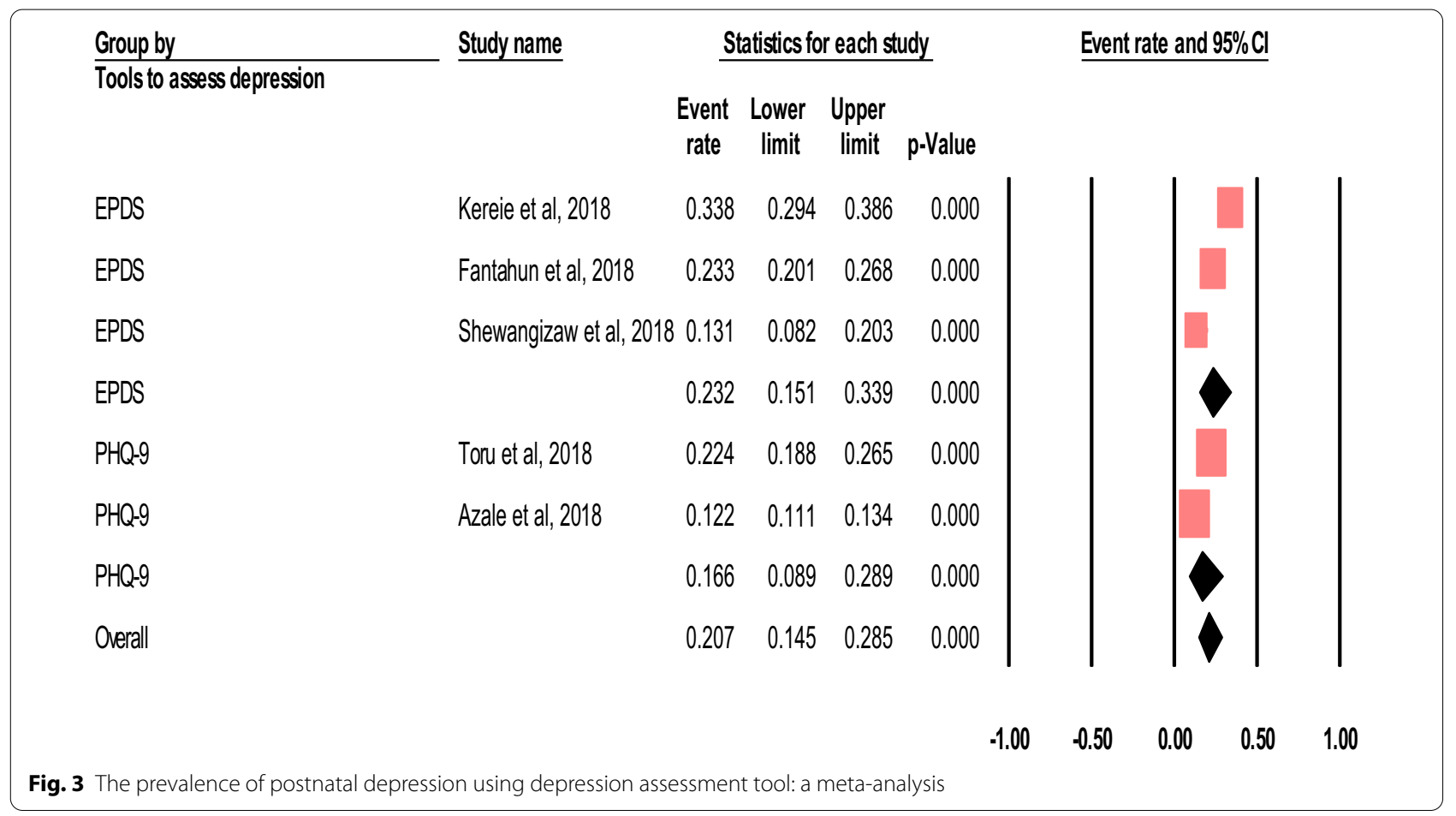

Table 2 Sensitivity analysis of prevalence of postnatal depression for each study being removed at a time: prevalence and $95 \%$ confidence

\begin{tabular}{lll}
\hline Study excluded & Prevalence & $\mathbf{9 5 \%} \mathbf{C l}$ \\
\hline Kereie et al. [11] & 15.4 & $14.3-16.5$ \\
Fantahun et al. [18] & 16.4 & $15.2-17.6$ \\
Toru et al. [14] & 16.8 & $15.7-18.0$ \\
Shewangizaw et al. [19] & 17.5 & $16.4-18.7$ \\
Azale et al. [20] & 25.4 & $23.3-27.6$ \\
\hline
\end{tabular}

The analysis is based on random effect model

and promote early screening and prevention of postnatal depression in Ethiopia.

The pooled prevalence estimate of this review was in line with studies conducted in South Africa [33, 34] and Nigeria [35]. However, the pooled estimated prevalence was higher than the studies' reports from Uganda [36] and Ghana [37] and lower than when compared to the reports from the study in South Africa [38]. The possible reasons for the observed difference in the prevalence of postnatal depression across the countries may be due to variations in socio-cultural and -economic factors, record keeping and reporting, and tools used to assess postnatal depression. For example, the studies conducted in South Africa and Uganda utilized Pitt depression inventory and Mini International

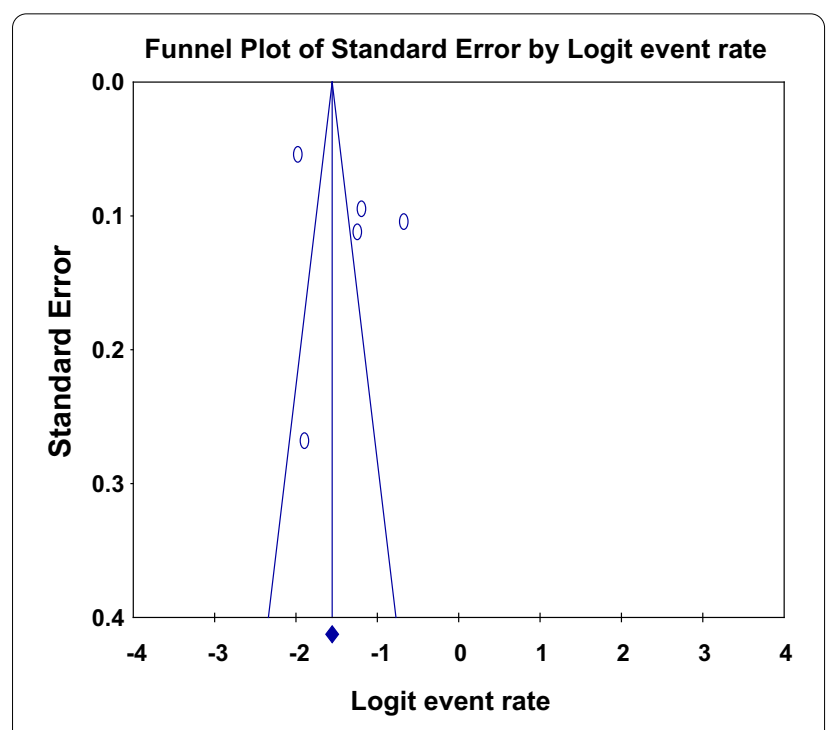

Fig. 4 Funnel plot showing no publication bias of included studies

Neuro-psychiatric Interview (MINI) respectively to assess postnatal depression. However, the studies included in our review used EPDS and PHQ9 only. Evidence from different studies also suggested that depressive symptoms in women increase in the first 2-12 weeks of delivery due to hormonal fluctuation and the new environment of childbirth [39, 40]. Thus, 
the interpretation of the estimates of this study should account for the window of measurement as a more precise window predicts more significant point estimates.

Unplanned pregnancy was associated with post-partum depression. In comparison to a planned pregnancy, women who did not plan their pregnancy were 3.46 times more likely to develop postnatal depression. This is in line with the studies conducted in Ghana and South Africa [41, 42]. An unplanned pregnancy may result from contraceptive failure and non-use of contraceptive services and, result in inadequate preparation for pregnancy and childbirth. This could exacerbate the physical health and psychological wellbeing of women and may result in postpartum depression. Further, women may develop postnatal depression due to stress which is associated with the transition into unplanned motherhood, suggesting strengthening the counseling services may contribute a significant role in alleviating a resultant depression. This is also complemented by a report from a prospective cohort study that suggested the minimal risk of depression was observed in women who planned their pregnancy [43].

In this review, women age between 15 and 24 years had 1.72 times the odds of having postnatal depression. The studies from Sudan also reported the same association [38]. This may be due to psychological distress as well as the stress of responsibility of marriage and childbirth. This is also supported by finding from a longitudinal study that reported the magnitude of depression was much higher among young people in their twenties [44].

We have also observed that women with marital problems such as divorced and widowed were prone to develop postnatal depression. Other studies also reported similar findings [44, 45]. However, the association between postnatal depression and divorce has not been explained yet. Nevertheless, some researchers hypothesized that depression by itself does not result in divorce and therefore, it is the result of not managing depression while others suggested that divorce alone can result in depression [46]. In contrast, a longitudinal study that used the data from the New Haven Epidemiologic Catchment Area (ECA) and investigated the association between marital problems and the risk of depressive episodes found a statistically significant association between marital disruption and the higher prevalence rates of depression in women [47].

The current review also found a high rate of postnatal depression among women experiencing the death of the infant. Findings from other studies in other countries also revealed a similar association. This may be due to losing an infant that can trigger intense feelings of grief and sadness. Having unmanaged feelings of grief and sadness after a loss could consequence postnatal depression. This is also supported by a cohort study that reported postnatal depression was significantly associated with approximately a three-fold increased risk of infant mortality [48].

Another important finding in the current review was that a remarkably higher rate of postnatal depression was observed among women with a history of a substance such as alcohol and khat use. A review of postpartum substance use and depression also supported this construct [49]. Different studies have been conducted so far revealed that women with postnatal depression are at a higher risk of developing substance abuse compared to women without postnatal depression.

\section{Limitations of the review}

A small number of studies were included in the review and this might over-or under-estimate the pooled estimated prevalence of postnatal depression in Ethiopia. The review also lacked a sub-group analysis using a region as a moderator due to the small number of studies.

\section{Conclusion}

The prevalence of postnatal depression in Ethiopia was high. Having unplanned pregnancy, age between 15 and 24 years, marital problems, experiencing death of infant, history of substance, poor social support, history of previous depression, domestic violence, grand multiparty, perinatal complications, experiencing hunger in the preceding 1 month, stressful events and history of abortion were factors significantly associated with postnatal depression. Therefore, the concerned body should give due attention to improve reproductive health services through early detection of risk factors of postnatal depression.

\section{Supplementary information}

Supplementary information accompanies this paper at https://doi. org/10.1186/s12978-020-01035-1.

Additional file 1: Epidemiology of postnatal depression in Ethiopia: a systematic review and meta-analysis (Pre-designed protocol).

\section{Abbreviations}

EPDS: Edinburgh Postnatal Depression Scale; LMICs: Low-income and middle-income countries; NOS: Newcastle Ottawa Scale; PHQ9: Patient Health Questionnaire.

\section{Acknowledgements}

No acknowledgments at this stage.

\section{Authors' contributions}

The author (BD) conceptualized the study, developed the manuscript, conducted the analyses, drafted, and approved the final manuscript. DW and YA performed the search, data extraction, and approved the final manuscript. All authors reviewed and approved the final manuscript.

Funding

The author declares that there is no funding. 


\section{Availability of data and material}

All data generated or analyzed during this study are included in this article.

\section{Ethics approval and consent to participate}

Not applicable.

\section{Consent for publication}

Not applicable.

\section{Competing interests}

The authors declare that there is no competing interests.

\section{Author details}

${ }^{1}$ Faculty of Heath Sciences, College of Medicine and Health Sciences, Hawassa University, Hawassa, Ethiopia. ${ }^{2}$ School of Public Health, Curtin University, Perth, Australia. ${ }^{3}$ Department of Medical Laboratory, Sodo Christian General Hospital, Wolaita Sodo, Ethiopia.

Received: 25 October 2019 Accepted: 1 November 2020 Published online: 19 November 2020

\section{References}

1. Stewart DE, Robertson E, Dennis C-L, Grace SL, Wallington T. Postpartum depression: literature review of risk factors and interventions. 2003. https ://www.who.int/mental_health/prevention/suicide/lit_review_postp artum depression.pdf. Accessed 20 Jan 2017.

2. Guille CN, Fryml R, et al. Management of postpartum depression. J Midwif Women's Health. 2013:58:643-53.

3. Chaudron LH, Chaudron M. Postpartum depression: what pediatricians need to know. Pediatr Rev. 2003;24(5):154-61.

4. World Health Organization. Fact sheet on depression. https://www.who. int/mental_health/management/depression/definition/en/.

5. World Health Organization, Depression and other common mental disorders: global health estimates. 2017.

6. GBD 2015 Disease and Injury Incidence and Prevalence Collaborators. Global, regional, and national incidence, prevalence, and years lived with disability for 310 diseases and injuries, 1990-2015: a systematic analysis for the Global Burden of Disease Study 2015. Lancet. 2016 Oct 8;388(10053):1545-602. https://doi.org/10.1016/S0140-6736(16)31678-6. (Erratum in: Lancet. 2017 Jan 7;389(10064):e1. PMID: 27733282; PMCID: PMC5055577).

7. Fisher J, de Cabral MM, Patel V, Rahman A, Tran T, Holton S, Holmes W. Prevalence and determinants of common perinatal mental disorders in women in low- and lower-middle-income countries: a systematic review. Bull World Health Organ. 2012;90(2):139G-G149.

8. Tsai AC, Tomlinson M, Comulada WS, Rotheram Borus MJ. Intimate partner violence and depression symptom severity among South African Women during pregnancy and postpartum: population-based prospective cohort study. PLoS Med. 2016;13(1):e1001943.

9. Rochat TJ, Tomlinson M, Barnighausen T, Newell M-L, Stein A. The prevalence and clinical presentation of antenatal depression in rural South Africa. J Affect Disord. 2011;135(1-3):362-73.

10. Heyningen TV, Myer L, Onah M, Tomlinson M, Field S, Honikman S. Antenatal depression and adversity in urban South Africa. J Affect Disord. 2016;203:121-9.

11. Kerie S, Menberu M, Niguse W. Prevalence and associated factors of postpartum depression in Southwest, Ethiopia, 2017: a cross-sectional study. BMC Res Notes. 2018;11(1):623.

12. Ali NS, Ali BS, Azam IS. Post partum anxiety and depression in peri-urban communities of Karachi, Pakistan: a quasi-experimental study. BMC Public Health. 2009;9(1):384

13. Tefera TB, Erena AN, Kuti KA, Hussen MA. Perinatal depression and associated factors among reproductive aged group women at Goba and Robe Town of Bale Zone, Oromia Region, South East Ethiopia. Matern Health Neonatol Perinatol. 2015:1:12

14. Toru T, Chemir F, Anand S. Magnitude of postpartum depression and associated factors among women in Mizan Aman town, Bench Maji zone, Southwest Ethiopia. BMC Pregnancy Childbirth. 2018;18(1):442.
15. Patel HL, Ganjiwale JD, Nimbalkar AS, Vani SN, Vasa R, Nimbalkar SM Characteristics of postpartum depression in Anand District, Gujarat. India J Trop Pediatr. 2015;61(5):364-9.

16. Turkcapar AF, Kadioglu N, Aslan E, Tunc S, Zayifoglu M, Mollamahmutoglu L. Sociodemographic and clinical features of postpartum depression among Turkish women: a prospective study. BMC Pregnancy Childbirth. 2015;15:108.

17. Stellenberg EL, Abrahams JM. Prevalence of and factors influencing postnatal depression in a rural community in South Africa. Afr J Prim Health Care Fam Med. 2015;7(1):1-8.

18. Fantahun A, Cherie A, Deribe L. Prevalence and factors associated with postpartum depression among mothers attending Public Health Centers of Addis Ababa, Ethiopia, 2016. Clin Pract Epidemiol Ment Health. 2018;14:196-206.

19. Abayneh S, Bilisuma T, Tiba A, Tadesse M, Sisay S. Prevalence of postpartum depression and associated factors among postnatal women attending At Hiwot Fana Specialized University Hospital, Harar, East Ethiopia, 2015/2016. Open Acc J Repro Sexual Disord 2018;1(1). OAJRSD. MS.ID.000102. https://doi.org/10.32474/OAJRSD.2018.01.000102

20. Azale T, Fekadu A, Hanlon C. Postpartum depressive symptoms in the context of high social adversity and reproductive health threats: a population-based study. Int J Ment Health Syst. 2018;12:42.

21. Gelaye $B$, et al. Epidemiology of maternal depression, risk factors, and child outcomes in low-income and middle-income countries. Lancet Psychiatry. 2016;3(10):973-82.

22. Stewart DE, et al. Postpartum depression: literature review of risk factors and interventions. Toronto: University Health Network Women's Health Program for Toronto Public Health; 2003.

23. Adewuya $\mathrm{AO}$, et al. Impact of postnatal depression on infants' growth in Nigeria. J Affect Disord. 2008;108(1-2):191-3.

24. Pendergast $L L$, et al. Postpartum depressive symptoms across time and place: structural invariance of the Self-Reporting Questionnaire among women from the international, multi-site MAL-ED study. J Affect Disord. 2014;167:178-86.

25. Sawyer A, Ayers S, Smith H. Pre-and postnatal psychological wellbeing in Africa: a systematic review. J Affect Disord. 2010;123(1-3):17-29.

26. Moher D, Shamseer L, Clarke M, Ghersi D, Liberati A, Petticrew M, et al. Group P-P: preferred reporting items for systematic review and metaanalysis protocols (PRISMA-P) 2015 statement. Syst Rev. 2015a;4(1):1.

27. Robertson E, Celasun N, Stewart D. Risk factors for postpartum depression. Geneva: World Health Organization; 2003.

28. Moher D, Shamseer L, Clarke M, Ghersi D, Liberati A, Petticrew M, et al. Preferred reporting items for systematic review and meta-analysis protocols (PRISMA-P) 2015 statement. Syst Rev. 2015b;4(1):1.

29. Stang A. Critical evaluation of the Newcastle-Ottawa scale for the assessment of the quality of nonrandomized studies in meta-analyses. Eur J Epidemiol. 2010;25(9):603-5.

30. Landis J, Koch G. The measurement of observer agreement for categorical data. Biometrics. 1977:33:159-74.

31. Borenstein M, Hedges LV, Higgins J, Rothstein HR. A basic introduction to fixed-effect and random-effects models for meta-analysis. Res Synth Methods. 2010;1(2):97-111.

32. Higgins JP, Thompson SG, Deeks JJ, Altman DG. Measuring inconsistency in meta-analyses. BMJ. 2003;327(7414):557.

33. Verkuijl NE, Richter L, Norris SA, Stein A, Avan B, Ramchandani PG. Postnatal depressive symptoms and child psychological development at 10 years: a prospective study of longitudinal data from the South African Birth to Twenty cohort. Lancet Psychiatry. 2014;1 (6):454-60

34. Mokhele I, Nattey C, Jinga N, Mongwenyana C, Fox MP, Onoya D. Prevalence and predictors of postpartum depression by HIV status and timing of HIV diagnosis in Gauteng, South Africa. PLOS ONE. 2019:14(4):e0214849.

35. Sulyman D, Ayanda K, Dattijo L, Aminu B. Postnatal depression and its associated factors among Northeastern Nigerian women. Ann Trop Med Public Health. 2016;9(3):184-90.

36. Nakku JE, Nakasi G, Mirembe F. Postpartum major depression at six weeks in primary health care: prevalence and associated factors. Afr Health Sci. 2006;6(4):207-14. https://doi.org/10.5555/afhs.2006.6.4.207.

37. Peltzer K, Shikwane M. Prevalence of postnatal depression and associated factors among HIV-positive women in primary care in Nkangala district, South Africa. South Afr J HIV Med. 2011;12(4):24. 
38. Stoltenberg EL, Abrahams JM. Prevalence of and factors influencing postnatal depression in a rural community in South Africa. Afr J Prm Health Care Fam Med. 2015;7(1):1-8.

39. BeckC T. State of the science on postpartum depression: what nurse researchers have contributed-part 2. MCN Am J Matern Child Nurs. 2008:33:151-6.

40. AffonsoD D, DeA K, Horowitz JA, Mayberry LJ. An international study exploring levels of postpartum depressive symptomatology. Psychosom Res. 2000:49(3):207-16.

41. Khalifa, et al. Postnatal depression among Sudanese women: prevalence and validation of the Edinburgh postnatal depression scale at 3 months postpartum. Int J Women's Health. 2015;7:677-84.

42. Juliet EM, Nakku GN, Florence M. Postpartum major depression at six weeks in primary health care: Prevalence and associated factors. Afr Health Sci. 2006;6(4):207-14.

43. Hall JA, Barrett G, Copas A, Phiri T, Malata A, Stephenson J. Reassessing pregnancy intention and its relation to maternal, perinatal and neonatal outcomes in a low-income setting: a cohort study. PLoS ONE. 2018;13(10):e0205487. https://doi.org/10.1371/journal.pone.0205487.

44. Gustavson K, Knudsen AK, Nesvåg R, et al. Prevalence and stability of mental disorders among young adults: findings from a longitudinal study. BMC Psychiatry. 2018;18:65. https://doi.org/10.1186/s12888-018-1647-5.
45. Wissart J, Parshad O, Kulkarni S. Prevalence of pre- and postpartum depression in Jamaican women. BMC Pregnancy Childbirth. 2005:5:15.

46. Zaidi F, Nigam A, Anjum R, Agarwalla R. Postpartum depression in women: a risk factor analysis. J Clin Diagn Res. 2017;11(8):QC13-6.

47. Bruce ML, Kim KM. Differences in the effects of divorce on major depression in men and women. Am J Psychiatry. 1992;149(7):914-7. https://doi. org/10.1176/ajp.149.7.914.

48. Weobong B, ten Asbroek AHA, Soremekun S, et al. Association between probable postnatal depression and increased infant mortality and morbidity: findings from the DON population-based cohort study in rural Ghana. BMJ Open. 2015;5:e006509. https://doi.org/10.1136/bmjop en-2014-006509.

49. Chapman SL, Wu LT. Postpartum substance use and depressive symptoms: a review. Women Health. 2013;53(5):479-503.

\section{Publisher's Note}

Springer Nature remains neutral with regard to jurisdictional claims in published maps and institutional affiliations.
Ready to submit your research? Choose BMC and benefit from:

- fast, convenient online submission

- thorough peer review by experienced researchers in your field

- rapid publication on acceptance

- support for research data, including large and complex data types

- gold Open Access which fosters wider collaboration and increased citations

- maximum visibility for your research: over $100 \mathrm{M}$ website views per year

At BMC, research is always in progress.

Learn more biomedcentral.com/submissions 